\title{
A Weighted Poisson Distribution for Underdispersed Count Data
}

\author{
Chedly Gélin Louzayadio ${ }^{1,4}$, Rodnellin Onésime Malouata ${ }^{2,4}$ \& Michel Diafouka Koukouatikissa ${ }^{3,4}$ \\ ${ }^{1}$ Faculty of Economic Sciences, Marien Ngouabi University, BP 69, Brazzaville, Congo \\ ${ }^{2}$ Higher Institute of Management, Marien Ngouabi University, BP 69, Brazzaville, Congo \\ ${ }^{3}$ Normal School Higher, Marien Ngouabi University, BP 69, Brazzaville, Congo \\ ${ }^{4}$ Laboratory of statistics and analysis of data (Labsad), Faculty of Sciences and Technics, Marien Ngouabi University, \\ BP 69, Brazzaville, Congo
}

Correspondence: Chedly Gélin Louzayadio, Faculty of Economic Sciences, Marien Ngouabi University, BP 69, Brazzaville, Congo. E-mail: gelinlouz@gmail.com

Received: March 1, 2021 Accepted: June 24, 2021 Online Published: June 28, 2021

doi:10.5539/ijsp.v10n4p157 URL: https://doi.org/10.5539/ijsp.v10n4p157

\begin{abstract}
In this paper, we present a new weighted Poisson distribution for modeling underdispersed count data. Weighted Poisson distribution occurs naturally in contexts where the probability that a particular observation of Poisson variable enters the sample gets multiplied by some non-negative weight function. Suppose a realization $y$ of $Y$ a Poisson random variable enters the investigator's record with probability proportional to $w(y)$ : Clearly, the recorded $y$ is not an observation on $Y$, but on the random variable $Y^{w}$, which is said to be the weighted version of $Y$. This distribution has three parameters and belongs to the exponential family, it includes and generalizes the Poisson distribution by weighting. It is a discrete distribution that is more flexible than other weighted Poisson distributions that have been proposed for modeling underdispersed count data, for example, the extended Poisson distribution (Dimitrov and Kolev, 2000). We present some moment properties and we estimate its parameters. One classical example is considered to compare the fits of this new distribution with the extended Poisson distribution.
\end{abstract}

Keywords: dispersion index, exponential family, Nelder-mead's algorithm, weighted Poisson distribution

AMS 2010 Mathematics subject classification: 62E15; 60E05

\section{Introduction}

The Poisson distribution is considered the standard distribution for the analysis of count data. However, it is equidispersed meaning that the mean is equal to the variance. Equivalently, the dispersion index (the ratio of variance to the mean), a measure of aggregation or repulsion, is always equal to one. In practice, the requirement that the index of dispersion should equal one is sometimes too restrictive. The data are often overdispersed, i.e. the dispersion index is greater than 1. For this purpose, a wade variety of distributions has been proposed to model the data. We can quote the negative binomial distribution, used since Greenwood and Yule in 1920, the weighted Poisson distribution proposed by Castillo and PérezCasany in 1998, the generalization of the Poisson distribution proposed by Consul in 1989, etc. More information on this topic is in Haight (1967), Johnson et al. (2005), Kendall and Stuart (1979).

The opposite phenomenon is underdispersion, where the index of dispersion is smaller than one. This phenomenon occurs less frequently, and the choice of distributions is much narrower (Ridout and Besbeas, 2004). Nevertheless, there are some situations in which underdispersion is well documented, (see, for example in Morgan, 1975 and 1982, Daley and Maindonald, 1989). However, to model the data, the distribution chosen does not always offer a better fit. It is, therefore, necessary to find another distribution that better describes the data. For example, data from Kendall in 1961 follow several distributions underdispersed in particular the extended Poisson distribution proposed by Dimitrov and Kolev in 2000.

The main goal of this paper is to propose another underdispersed distribution that better describes Kendall's data and to compare it with the extended Poisson distribution. The distribution proposed here is a weighted Poisson distribution with three parameters, $\theta>0, \gamma>\frac{2}{9}$ and $\xi>1$; we note it $\operatorname{WPD}(\theta, \gamma, \xi)$. The weighted Poisson distribution is an alternative to the Poisson distribution when overdispersion or underdispersion is present (Patil and Rao, 1978, Patil 2002). It allows us to take into account, among others, the phenomenon of dispersion; we can refer, for example, to Gupta \& Ong in 2005, Shmueli in 2005, Louzayadio et al. in 2013, Louzayadio in 2015, Mizère et al. in 2006, 2013 and their references. A more general procedure to obtain the weighted Poisson distribution is to multiply the Poisson distribution by the ratio of weight function to the normalizing constant; (Kokonendji et al., 2008) and their references as well as Balakrishnan \& Kozubowski 
in 2008. The weight function that usually appears in the scientific and statistical literature is $w(y)=y$, which provides the size-biased version of the random variable. The size-biased version of order $k$, which corresponds to the weight $w(y)=y^{k}$ and $w(y)=(y+a)^{k}$, where $k$ is a real positive number and $a$ is a positive displacement parameter, have also been widely used (see, Castillo \& Pérez-Casany, 1998). The weight function proposed in this paper is $w_{\gamma, \xi}(y)=1+\gamma\left(y^{2}+2 y-1 / \xi\right)$.

This article is organized as follows: Section 2 reviews and presents some definitions and properties of weighted Poisson distributions. In Section 3, we introduce the new weighted Poisson distribution WPD $(\theta, \gamma, \xi)$ and we discuss some of its important features and properties such as its mass function, its cumulative distribution function, its moment generating function, its moments, its index of dispersion, and its entropy. In Section 4, we compare the stochastic order relationship between two random variables $\operatorname{WPD}(\theta, \gamma, \xi)$. In section 5 , we estimate parameters of $\operatorname{WPD}(\theta, \gamma, \xi)$. Finally, in Section 6 , we compare the weighted Poisson distribution $\operatorname{WPD}(\theta, \gamma, \xi)$ and the extended Poisson distribution $\operatorname{EPD}(\theta, \beta)$ using real data.

\section{Preliminaries}

In this section, we recall and propose some definitions and properties of weighted Poisson distributions.

Definition 1 (Castillo and Pérez-Casany, 1998) The distribution of Castillo and Pérez-Casany is defined by

$$
p^{w}(y ; \theta, \phi)=\frac{(y+a)^{r}}{\sum_{j=0}^{+\infty}(j+a)^{r} \frac{\theta^{j}}{j !}} \frac{\theta^{y}}{y !} e^{-\theta}, \quad y \in \mathbb{N}, a>0, r>0 \quad \text { and } \quad \phi=(a, r) .
$$

It is a weighted Poisson distribution of weight function

$$
w(y, \phi)=(y+a)^{r}
$$

and normalizing constant

$$
E_{\theta}[w(Y, \phi)]=\sum_{j=0}^{+\infty}(j+a)^{r} \frac{\theta^{j}}{j !} e^{-\theta}
$$

finite.

Definition 2 (Dimitrov and Kolev, 2000) Let $Y$ be a $\beta$-transformation of a Poisson random variable $X$ with mean $\theta>0$. We call extended Poisson distribution denoted by $\operatorname{EPD}(\theta, \beta)$, the distribution of the random variable $Y$ defined by

$$
P(Y=y)=\left\{\begin{array}{ll}
\left(1-e^{-\theta}\right) / \beta & y=0 \\
\frac{e^{-\theta} \theta^{y}}{\beta y !}\left(\frac{\beta y}{\theta}-1\right) & y=1,2, \ldots
\end{array} \quad ; \forall \theta>0, \beta \in\right] 0,1[.
$$

We can write this probability mass function (pmf) in the following way

$$
P\left(Y^{w}=y\right)=\frac{\theta^{y}}{y !} e^{-\theta}\left(\frac{\beta}{\theta} y-1\right) \beta^{-1}\left\{\frac{1-e^{-\theta}}{\left(\frac{\beta}{\theta} y-1\right) e^{-\theta}}\right\}^{\delta_{0}(y)} ;
$$

where

$$
\delta_{0}(y)=\left\{\begin{array}{ccc}
1 & \text { si } & y=0 \\
0 & \text { else }
\end{array}\right.
$$

is the indicator function in 0.

It is a weighted Poisson distribution of weight function

$$
w(y, \theta, \beta)=\left(\frac{\beta}{\theta} y-1\right)\left\{\frac{1-e^{-\theta}}{\left(\frac{\beta}{\theta} y-1\right) e^{-\theta}}\right\}^{\delta_{0}(y)}
$$

and normalizing constant

$$
E_{\theta}[w(Y, \phi)]=\beta .
$$

The weighted version $Y^{w}$ of $Y$ is characterized by:

$$
E\left(Y^{w}\right)=1+\frac{\beta-1}{\beta} \theta \text { and } V\left(Y^{w}\right)=\frac{\beta-1}{\beta^{2}} \theta^{2}+\frac{\beta+1}{\beta} \theta .
$$


If $\beta>\theta$ with $\beta \in] 0,1\left[\right.$, the $\operatorname{EPD}(\theta, \beta)$ is underdispersed because, its index of dispersion $I_{Y^{w}}(\theta)<1$.

Kokonendji et al. in 2008 found several links between weight function and overdispersion or underdispersion. More specifically, they proved that the log-convexity (log-concavity) of the mean weight function is a necessary and sufficient condition for a weighted version of a Poisson variable to be overdispersed (underdispersed). We present their results in the following two proposals:

Proposition 1 Let $Y$ be a Poisson random variable with mean $\theta>0$ and let

$$
w(y)=w(y ; \phi), y \in \mathbb{N},
$$

be a weight function not depending on $\theta$. Then, the mean weight function $\theta \longmapsto E_{\theta}[w(Y)]$ is log-convex (or log-concave) if and only if the weighted version $Y^{w}$ of $Y$ is overdispersed ( or underdispersed). On the other side, the following proposition allows us to prove that the index of dispersion of $\operatorname{WPD}(\theta, \gamma, \xi)$ (see section 3 ) is smaller than one.

Proposition 2 Let $Y$ be a Poisson random variable with mean $\theta>0$ and let

$$
w(y)=w(y ; \phi), y \in \mathbb{N},
$$

be a weight function not depending on the Poisson mean parameter $\theta$. Then,

$$
\operatorname{Var}_{\theta}\left(Y^{w}\right)=E_{\theta}\left(Y^{w}\right)+\theta^{2} \frac{d^{2}}{d \theta^{2}} \ln \left[E_{\theta}(w(Y ; \phi))\right] .
$$

\section{Another Weighted Poisson Distribution and Base Properties}

Let $Y$ be a standard Poisson random variable with probability mass function (pmf)

$$
p(y, \theta)=\frac{\theta^{y}}{y !} e^{-\theta}, \quad y \in \mathbb{N}, \quad \theta>0 ;
$$

where $\theta$ is the canonical parameter. We consider for $\gamma>\frac{2}{9}$ and $\xi>1$, the weight function

$$
w_{\gamma, \xi}(y)=1+\gamma\left(y^{2}+2 y-1 / \xi\right),
$$

the probability mass function (pmf) of the weighted version $Y^{w_{\gamma, \xi}}$ of $Y$ given by:

$$
p^{w_{\gamma, \xi}}(y, \theta)=\frac{w_{\gamma, \xi}(y)}{E_{\theta}\left[w_{\gamma, \xi}(Y)\right]} p(y, \theta), \quad y \in \mathbb{N}
$$

is a weighted Poisson distribution, denoted by WPD $(\theta, \gamma, \xi)$ where, $E_{\theta}\left[w_{\gamma, \xi}(Y)\right]$ is the normalizing constant.

Let us remark that, we can write (5) in the following way

$$
p^{w_{\gamma, \xi}}(y, \theta)=\frac{\exp \left(y \ln \theta+\ln \left[w_{\gamma, \xi}(y)\right]\right)}{y ! K(\theta, \gamma, \xi)},
$$

where

$$
K(\theta, \gamma, \xi)=\left[1+\gamma\left(\theta^{2}+3 \theta-1 / \xi\right)\right] e^{\theta} .
$$

From (6) we see that the WPD $(\theta, \gamma, \xi)$ is an element of the natural exponential family on $\mathbb{N}$. In the figure 1 shown with the software $\mathrm{R}$ we show the appearance of the new weighted Poisson distribution.

The cumulative distribution function (cdf) of a random variable $Y^{w_{\gamma, \xi}}$ following a WPD $(\theta, \gamma, \xi)$ distribution is given by

$$
F_{Y^{w}, \xi}=E_{\theta}\left[w_{\gamma, \xi}(Y)\right]-\theta^{y} E_{\theta}\left[\frac{t !}{(Y+t) !} w_{\gamma, \xi}(Y+t)\right]
$$

where $t \in \mathbb{N}$.

Through the following propositions, we give the characteristics of $\operatorname{WPD}(\theta, \gamma, \xi)$.

Proposition 3 Let $Y^{w_{\gamma, \xi}}$ be a WPD $(\theta, \gamma, \xi)$ random variable. The moments generating function of $Y^{w_{\gamma, \xi},} M_{Y^{w_{\gamma, \xi}}}(t)$, is given by

$$
M_{Y^{w_{\gamma, \xi}}}(t)=\frac{\exp \left[\theta\left(e^{t}-1\right)\right]}{1+\gamma\left(\theta^{2}+3 \theta-\frac{1}{\xi}\right)}\left(1+\theta^{2} \gamma e^{2 t}+3 \theta \gamma e^{t}-\frac{\gamma}{\xi}\right) .
$$




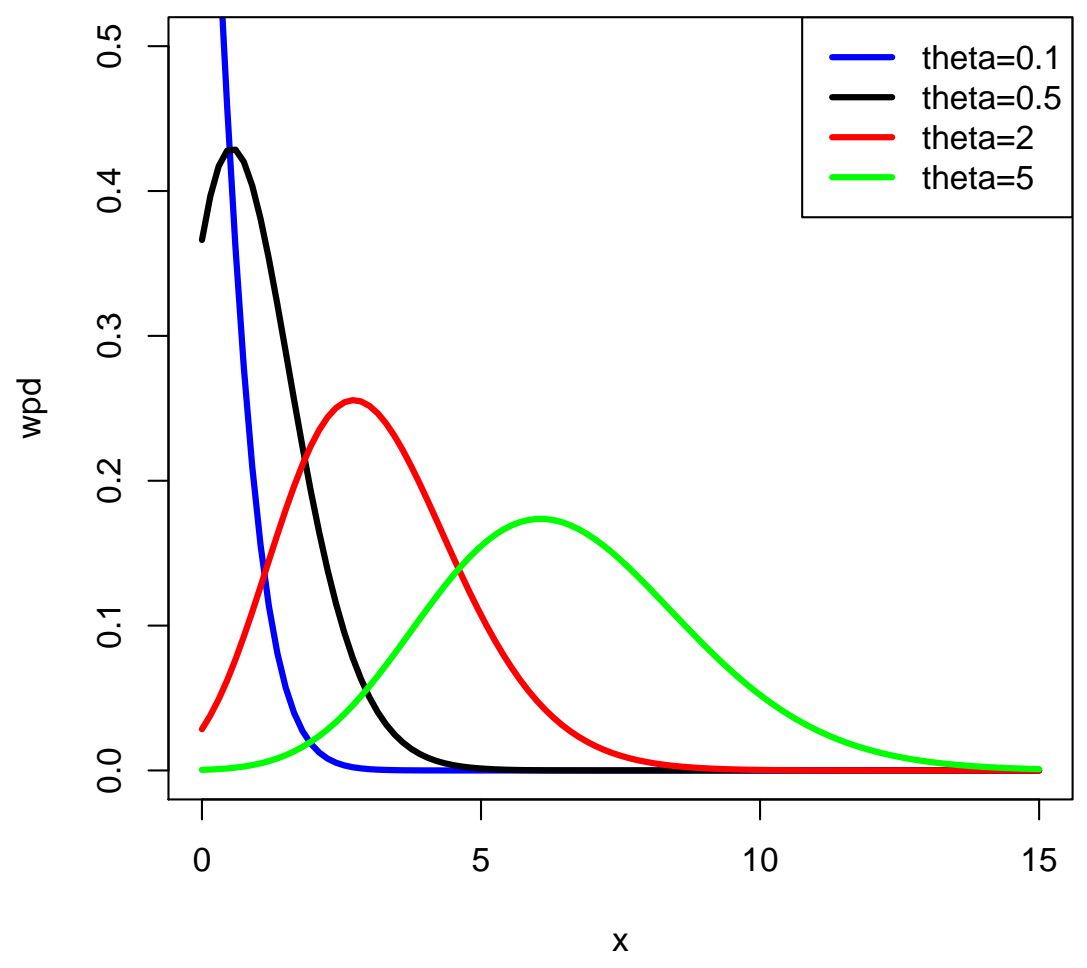

Figure 1. Probability mass functions (6) for $\theta=0.1,0.5,2,5, \gamma=0.3$ and $\xi=1.5$

\section{Proof.}

$$
\begin{aligned}
M_{Y^{w_{\gamma, \xi}}}(t) & =E\left(e^{t Y^{w_{\gamma, \xi}}}\right)=\sum_{y=0}^{+\infty} e^{t y} p^{w_{\gamma, \xi}}(y, \theta) \\
& =\frac{e^{-\theta}}{1+\gamma\left(\theta^{2}+3 \theta-\frac{1}{\xi}\right)} \sum_{y=0}^{+\infty}\left(e^{t y}\left[1+\gamma\left(y^{2}+2 y-\frac{1}{\xi}\right)\right] \frac{\theta^{y}}{y !}\right) \\
& =\frac{e^{-\theta}}{1+\gamma\left(\theta^{2}+3 \theta-\frac{1}{\xi}\right)}\left(1+\theta^{2} \gamma e^{2 t}+3 \theta \gamma e^{t}-\frac{\gamma}{\xi}\right) \sum_{y=0}^{+\infty} \frac{\left(\theta e^{t}\right)^{y}}{y !} \\
& =\frac{\exp \left[\theta\left(e^{t}-1\right)\right]}{1+\gamma\left(\theta^{2}+3 \theta-\frac{1}{\xi}\right)}\left(1+\theta^{2} \gamma e^{2 t}+3 \theta \gamma e^{t}-\frac{\gamma}{\xi}\right)
\end{aligned}
$$

Proposition 4 If $Y^{w_{\gamma, \xi}}$ is a $\operatorname{WPD}(\theta, \gamma, \xi)$ random variables, then we have

$$
E\left(Y^{w_{\gamma, \xi}}\right)=\frac{d}{d \mu} b(\mu)=\gamma \frac{\theta^{3}+5 \theta^{2}+\left(3+\frac{1}{\gamma}-\frac{1}{\xi}\right) \theta}{1+\gamma\left(\theta^{2}+3 \theta-\frac{1}{\xi}\right)},
$$


and

$$
\operatorname{Var}\left(Y^{w_{\gamma, \xi}}\right)=\gamma\left\{\frac{\gamma \theta^{4}+6 \gamma \theta^{3}+\left(12 \gamma^{2}+\frac{\gamma^{2}}{\xi}-\gamma-\frac{3}{\xi} \gamma+3\right) \theta^{2}+10\left(\gamma-\frac{\gamma^{2}}{\xi}\right) \theta+\frac{\gamma^{2}}{\xi^{2}}-3 \frac{\gamma^{2}}{\xi}+3 \gamma-2 \frac{\gamma}{\xi}+1}{\left[1+\gamma\left(\theta^{2}+3 \theta-\frac{1}{\xi}\right)\right]^{2}}\right\}
$$

Proof. In fact, the $\operatorname{WPD}(\theta, \gamma, \xi)$ is an element of the natural exponential family, because

$$
p^{w_{\gamma, \xi}}(y, \theta)=\frac{1+\gamma\left(y^{2}+2 y-\frac{1}{\xi}\right)}{y !} \exp \left\{y \ln \theta-\ln \left[1+\gamma\left(\theta^{2}+3 \theta-\frac{1}{\xi}\right)\right]-\theta\right\} ;
$$

with canonical parameter and cumulant function respectively

$$
\mu=\ln \theta \text { and } b(\mu)=\ln \left[1+\gamma\left(e^{2 \mu}+3 e^{\mu}-\frac{1}{\xi}\right)\right]+e^{\mu},
$$

(Jørgensen, 1997). So, the mean is

$$
E\left(Y^{w_{\gamma, \xi}}\right)=\frac{d}{d \mu} b(\mu)=\gamma \frac{e^{3 \mu}+5 e^{2 \mu}+\left(3+\frac{\xi-\gamma}{\gamma \xi}\right) e^{\mu}}{1+\gamma\left(e^{2 \mu}+3 e^{\mu}-\frac{1}{\xi}\right)} ;
$$

by replacing $e^{\mu}$ with $\theta$ we have the result. Taking the derivative of the mean $E\left(Y^{w_{\gamma, \xi}}\right)$ with respect to $\theta$, we obtain the variance.

Proposition 5 Let $Y^{w_{\gamma, \xi}}$ be a $\operatorname{WPD}(\theta, \gamma, \xi)$ random variable. The entropy of $P^{w_{\gamma, \xi}}$ noted $H\left(P^{w_{\gamma, \xi}}\right)$, is given by

$$
H\left(P^{w_{\gamma, \xi}}\right)=\ln \left(E_{\theta}\left[w_{\gamma, \xi}(Y)\right]\right)+\frac{E_{\theta}\left[w_{\gamma, \xi}(Y) \ln (Y !)\right]}{E_{\theta}\left[w_{\gamma, \xi}(Y)\right]}-\theta \ln \left[\theta\left(1+\frac{2 \theta+3-1 / \xi}{E_{\theta}\left[w_{\gamma, \xi}(Y)\right]}\right)\right]-\frac{E_{\theta}\left[w_{\gamma, \xi}(Y) \ln \left(w_{\gamma, \xi}(Y)\right)\right]}{E_{\theta}\left[w_{\gamma, \xi}(Y)\right]}+\theta .
$$

\section{Proof.}

$$
\begin{aligned}
H\left(P^{\left.w_{\gamma, \xi}\right)}=\right. & -\sum_{y=0}^{+\infty} \ln \left(P^{w_{\gamma, \xi}}(y, \theta)\right) P^{w_{\gamma, \xi}}(y, \theta) \\
= & -\sum_{y=0}^{+\infty}\left(\left\{\ln \left[1+\gamma\left(y^{2}+2 y-1 / \xi\right)\right]-\ln \left[1+\gamma\left(\theta^{2}+3 \theta-1 / \xi\right)\right]+\ln \left(\frac{\theta^{y}}{y !}\right)-\theta\right\}\right. \\
& \left.\left\{\frac{1+\gamma\left(y^{2}+2 y-1 / \xi\right)}{1+\gamma\left(\theta^{2}+3 \theta-1 / \xi\right)} \frac{\theta^{y}}{y !} e^{-\theta}\right\}\right) \\
= & -\sum_{y=0}^{+\infty}\left(\left\{\frac{\ln \left[1+\gamma\left(y^{2}+2 y-1 / \xi\right)\right]\left(1+\gamma\left(y^{2}+2 y-1 / \xi\right)\right)}{1+\gamma\left(\theta^{2}+3 \theta-1 / \xi\right)}\right\} \frac{\theta^{y}}{y !} e^{-\theta}\right) \\
& +\sum_{y=0}^{+\infty}\left(\left\{\frac{\ln \left[1+\gamma\left(\theta^{2}+3 \theta-1 / \xi\right)\right]\left(1+\gamma\left(y^{2}+2 y-1 / \xi\right)\right)}{1+\gamma\left(\theta^{2}+3 \theta-1 / \xi\right)}\right\} \frac{\theta^{y}}{y !} e^{-\theta}\right) \\
& -\sum_{y=0}^{+\infty}\left(\left\{\frac{\ln \left[1+\gamma\left(y^{2}+2 y-1 / \xi\right)\right]}{1+\gamma\left(\theta^{2}+3 \theta-1 / \xi\right)} y \ln \theta\right\} \frac{\theta^{y}}{y !} e^{-\theta}\right) \\
& +\sum_{y=0}^{+\infty}\left(\left\{\frac{1+\gamma\left(y^{2}+2 y-1 / \xi\right)}{1+\gamma\left(\theta^{2}+3 \theta-1 / \xi\right)} \ln (y !)\right\} \frac{\theta^{y}}{y !} e^{-\theta}\right)+\theta \\
= & \ln \left(E_{\theta}\left[w_{\gamma, \xi}(Y)\right]\right)+\frac{E_{\theta}\left[w_{\gamma, \xi}(Y) \ln (Y !)\right]}{E_{\theta}\left[w_{\gamma, \xi}(Y)\right]}-\theta \ln \theta\left(1+\frac{2 \theta+3-1 / \xi}{E_{\theta}\left[w_{\gamma, \xi}(Y)\right]}\right) \\
& -\frac{E_{\theta}\left[w_{\gamma, \xi}(Y) \ln \left(w_{\gamma, \xi}(Y)\right)\right]}{E_{\theta}\left[w_{\gamma, \xi}(Y)\right]}+\theta . \\
&
\end{aligned}
$$


Proposition 6 If $\gamma=1$ and $\xi$ tends to infinity, then the WPD $(\theta, \gamma, \xi)$ random variable $Y^{w_{\gamma, \xi}}$ converges in distribution to a weighted Poisson distribution random variable $Y^{w}$ of Castillo and Pérez-Casany proposed in 1998 for $a=1$ and $r=2$.

Proof.

$$
F_{1, \xi}(y)=\sum_{k \leq y} p^{w_{1, \xi}}(k, \theta)=\sum_{k=0}^{\min ([y], \xi)} \frac{1+k^{2}+2 k-1 / \xi}{1+\theta^{2}+3 \theta-1 / \xi} \frac{\theta^{k}}{k !} e^{-\theta}
$$

where $[y]$ denotes the integer part of $y$. For $\xi \geq[y]$,

$$
F_{1, \xi}(y)=\sum_{k=0}^{[y]} p^{w_{1, \xi}}(k, \theta)
$$

When $\xi$ tends to infinity, $F_{1, \xi}(y)$ tends to the weighted Poisson distribution introduced by Castillo and Pérez-Casany in 1998 , for $a=1$ and $r=2$. Hence, the proposition.

Proposition 7 The $\operatorname{WPD}(\theta, \gamma, \xi)$ random variables $Y^{w_{\gamma, \xi}}$ is underdispersed.

Proof. Taking the logarithm of the normalizing constant we have

$$
\ln E_{\theta}\left[w_{\gamma, \xi}(Y)\right]=\ln \left[1+\gamma\left(\theta^{2}+3 \theta-\frac{1}{\xi}\right)\right] .
$$

Now, differentiating with respect to $\theta$ we obtain

$$
\frac{d \ln E_{\theta}\left[w_{\gamma, \xi}(Y)\right]}{d \theta}=\gamma \frac{2 \theta+3}{1+\gamma\left(\theta^{2}+3 \theta-\frac{1}{\xi}\right)}
$$

and,

$$
\frac{d^{2} \ln E_{\theta}\left[w_{\gamma, \xi}(Y)\right]}{d \theta^{2}}=-\gamma^{2} \frac{2 \theta^{2}+6 \theta+2 \frac{\gamma-\xi}{\gamma \xi}+9}{\left[1+\gamma\left(\theta^{2}+3 \theta-\frac{1}{\xi}\right)\right]^{2}}<0
$$

then, proposition 1 ensures the result.

Corolary 1 If $Y^{w_{\gamma, \xi}}$ is a WPD $(\theta, \gamma, \xi)$ random variables, then its index of dispersion, $I_{Y^{w_{\gamma}, \xi}}(\theta)$ is such that:

$$
I_{Y^{w_{\gamma} \xi}}(\theta)=\frac{\gamma \theta^{4}+6 \gamma \theta^{3}+\left(12 \gamma^{2}+\frac{\gamma^{2}}{\xi}-\gamma-\frac{3}{\xi} \gamma+3\right) \theta^{2}+10\left(\gamma-\frac{\gamma^{2}}{\xi}\right) \theta+\frac{\gamma^{2}}{\xi^{2}}-3 \frac{\gamma^{2}}{\xi}+3 \gamma-2 \frac{\gamma}{\xi}+1}{\left[\theta^{3}+5 \theta^{2}+\left(3+\frac{1}{\gamma}-\frac{1}{\xi}\right) \theta\right]\left[1+\gamma\left(\theta^{2}+3 \theta-\frac{1}{\xi}\right)\right]}<1 .
$$

Proof. In fact,

$$
I_{Y^{w_{\gamma, \xi}}}(\theta)=\frac{V\left(Y^{w_{\gamma, \xi}}\right)}{E\left(Y^{w_{\gamma, \xi}}\right)}
$$

The propositions 1, 2 and 4 ensures the result.

\section{Stochastic Order Relations}

In this section, we are going to compare the stochastic order relation between two WPD random variables. If the weight function $w$ is increasing, (Patil et al., 1986) proved that the weighted version $Y^{w}$ of a random variable $Y$ stochastically dominates the original random variable $Y$. Since the weight function $w_{\gamma, \xi}(y)$ of WPD $(\theta, \gamma, \xi)$ is increasing in then the weighted version $Y^{w_{\gamma, \xi}}$ stochastically dominates the Poisson random variable $Y$.

This result allows us to establish the following proposition.

Proposition 8 Let $w_{\gamma, \xi}$ be an increasing function. For $i=1,2$, let $Y_{i}^{w_{\gamma_{i}, \xi_{i}}}$ be a $\operatorname{WPD}\left(\theta_{i}, \gamma_{i}, \xi_{i}\right)$ random variables. If $\theta_{1} \leq \theta_{2}$, $\gamma_{1}=\gamma_{2}$ and $\xi_{1} \leq \xi_{2}$ (ou $\left.\xi_{1}=\xi_{2}\right)$ then, $Y_{2}^{w_{\gamma, \xi}}$ stochastically dominates $Y_{1}^{w_{\gamma, \xi}}$. If $\gamma_{1} \leq \gamma_{2}, \theta_{1}=\theta_{2}$ and $\xi_{1}=\xi_{2}$ then, $Y_{2}^{w_{\gamma, \xi}}$ stochastically dominates $Y_{1}^{w_{\gamma, \xi}}$. If $\xi_{1} \leq \xi_{2}, \gamma_{1}=\gamma_{2}$ and $\theta_{1}=\theta_{2}\left(\right.$ ou $\left.\theta_{1} \leq \theta_{2}\right)$ then, $Y_{2}^{w_{\gamma, \xi}}$ stochastically dominates $Y_{1}^{w_{\gamma, \xi}}$.

Proof. Let us suppose that $\theta_{1} \leq \theta_{2}, \gamma_{1}=\gamma_{2}$ and $\xi_{1} \leq \xi_{2}$ (ou $\left.\xi_{1}=\xi_{2}\right)$ then $E_{\theta_{1}}\left[w_{\gamma, \xi}\left(Y_{1}\right)\right] \leq E_{\theta_{2}}\left[w_{\gamma, \xi}\left(Y_{2}\right)\right]$. We have the result, because the weight function $w_{\gamma, \xi}($ ) is increasing (Saporta, 2006). Similarly, we prove the others cases. 


\section{Estimation}

In this section, we will estimate the parameters $\theta, \gamma$ and $\xi$ by using the maximum likelihood method. We denote by $\widehat{\theta}$, $\widehat{\gamma}$, and $\widehat{\xi}$, the maximum likelihood estimators of the parameters $\theta, \gamma$ and $\xi$ respectively. Let $\left(y_{1}, y_{2}, \ldots, y_{n}\right)$ be a random sample of $n$ size of $\operatorname{WPD}(\theta, \gamma, \xi)$; then the log-likelihood function $L(y, \theta, \gamma, \xi)$ is

$$
\begin{aligned}
L(y, \theta, \gamma, \xi)= & \sum_{i=1}^{n} \ln \left[1+\gamma\left(y_{i}^{2}+2 y_{i}-\frac{1}{\xi}\right)\right]-n \bar{y} \ln (\theta)-n \theta-\sum_{i=1}^{n} \ln \left(y_{i} !\right) \\
& -n \ln \left[1+\gamma\left(\theta^{2}+3 \theta-\frac{1}{\xi}\right)\right] .
\end{aligned}
$$

Taking the partial derivative of the $\log$-likelihood function with respect to $\theta, \gamma$ and $\xi$ and equaling to zero respectively, we obtain the following equations:

$$
\begin{gathered}
\frac{\partial L}{\partial \theta}=-\frac{n \bar{y}}{\theta}-n-n \frac{2 \gamma \theta+3 \gamma}{1+\gamma\left(\theta^{2}+3 \theta-\frac{1}{\xi}\right)}=0 \\
\frac{\partial L}{\partial \gamma}=\sum_{i=1}^{n}\left(\frac{y_{i}^{2}+2 y_{i}-\frac{1}{\xi}}{1+\gamma\left(y_{i}^{2}+2 y_{i}-\frac{1}{\xi}\right)}\right)-n \frac{\theta^{2}+3 \theta-\frac{1}{\xi}}{1+\gamma\left(\theta^{2}+3 \theta-\frac{1}{\xi}\right)}=0 \\
\frac{\partial L}{\partial \xi}=\frac{\gamma}{\xi^{2}} \sum_{i=1}^{n}\left(\frac{1}{1+\gamma\left(y_{i}^{2}+2 y_{i}-\frac{1}{\xi}\right)}\right)-\frac{n \gamma}{\xi^{2}} \frac{1}{1+\gamma\left(\theta^{2}+3 \theta-\frac{1}{\xi}\right)}=0 .
\end{gathered}
$$

These equations are non-linear, we use the Nelder Mead algorithm (Nelder and Mead, 1965) implemented in the R fitdistplus package to solve them simultaneously.

\section{Application}

In this section, we give an example of fitting practical data by the WPD $(\theta, \gamma, \xi)$. In this example, we compare the results with the fits given by the weighted Poisson distribution WPD $(\theta, \gamma, \xi)$ and the extended Poisson distribution EPD $(\theta, \beta)$. The statistical data (see, table 1) are taken from Kendall (1961) and correspond to the observed data on the number of outbreaks of strikes in 4-week periods, in a coal mining industry in the United Kingdom during 1948-1959. These observations are modestly underdispersed because the ratio of the sample variance $s^{\prime 2}=0.741894$ to the sample mean $\bar{y}=0.99359$ is smaller than one. The different observed and expected values are computed by the $\chi^{2}$ test of Pearson.

The column called $P(\theta)$ of Table 1 contains the corresponding expected frequencies calculated by using the Poisson distribution with parameter $\theta$, whose estimate is $\widehat{\theta}=\bar{y}=0.99359$. The values of the Chi-square and the p-value respectively, for the goodness of fit test for this distribution are $\chi^{2}=10.492$ and $p$-value $=0.015$ respectively.The value of the khi-two is too high, so the insufficiency of the Poisson distribution to the data is obvious. The reason for this is that the variance in the sample is lower than the sample average, whereas it should be almost equal.

The column EPD $(\theta, \beta)$ of table 1 contains the corresponding expected frequencies calculated by using the Extended Poisson distribution. The maximum likelihood estimates of the $\theta$ and $\beta$ parameters are

$$
\widehat{\theta}_{M L}=0.3417 \text { and } \widehat{\beta}_{M L}=0.9947 \text {. }
$$

The value of chi-square for $\operatorname{EPD}(\theta, \beta)$, for instance, is $\chi^{2}=3.970$ and the corresponding p-value is 0.265 . Thus, we cannot reject the idea that the data come from the Extended Poisson distribution at the usual significance level $\alpha=0.05$.

In this paper, we do not discuss the maximum likelihood estimates of the $\theta, \gamma$ and $\xi$ parameters of the $\operatorname{WPD}(\theta, \gamma, \xi)$ and their properties. The corresponding results are given here for comparison only. The maximum likelihood estimates of the $\theta, \gamma$ and $\xi$ parameters are obtained numerically because the log-likelihood function is non-linear. We based ourselves on the optimization method of Nelder Mead's algorithm. We obtained the following maximum likelihood estimates

$$
\widehat{\theta}_{M L}=0.3567569, \widehat{\gamma}_{M L}=0.7733672 \text { et } \widehat{\xi}_{M L}=2.4390914 \text {. }
$$

The value of the chi-square for the goodness of the fit test for the $\operatorname{WPD}(\theta, \gamma, \xi)$ with the above estimated parameters is $\chi^{2}=2.398$ and the $\mathrm{p}$-value $=0.663$. Thus, fit of the observed data by the $\operatorname{WPD}(\theta, \gamma, \xi)$, even with parameters estimated by 
the optimization method of Nelder Mead's algorithm, is acceptable, while the original Poisson model is not it. However, a comparison of the expected values present in the columns $\operatorname{EPD}(\theta, \beta)$ and WPD $(\theta, \gamma, \xi)$ of table 1 shows that the $\mathrm{WPD}(\theta, \gamma, \xi)$ offers better goodness of fit to statistical data than the $\operatorname{EPD}(\theta, \beta)$.

Table 1. Model fits to the number of outbreaks of strikes (M.G. Kendall, 1961)

\begin{tabular}{lcccc}
\hline $\mathrm{y}$ & Frequencies observed & $\mathrm{P}(\theta)$ & $\mathrm{EPD}(\theta, \beta)$ & $\mathrm{WPD}(\theta, \gamma, \xi)$ \\
\hline 0 & 46 & 57.758 & 45.393 & 46.343 \\
1 & 76 & 57.388 & 72.769 & 72.702 \\
2 & 24 & 28.510 & 31.371 & 29.667 \\
3 & 9 & 9.442 & 5.730 & 6.308 \\
$\geq 4$ & 1 & 2.902 & 0.221 & 0.881 \\
& $\chi^{2}$ & 10.492 & 3.970 & 2.398 \\
& $\mathrm{p}$-value & 0.015 & 0.265 & 0.663 \\
\hline
\end{tabular}

\section{Conclusion}

In this article, we have proposed a new generalization by weighting of the Poisson distribution. Some important basic properties and the problem of the estimation of its parameters have been studied. We have shown that this new weighted Poisson distribution can be used to model the under dispersed count data, for example, data from Kendall (1961).

\section{References}

Balakrishnan, N., \& Kozubowski, T. J. (2008). A class of weighted Poisson processes. Statistics $\mathcal{E}$ Probability Letters 78, 2346-2352. https://doi.org/10.1016/j.spl.2008.02.011

Castillo, J. D., \& Pérez-Casany, M. (1998). Weighted Poisson distributions for overdispersion and underdispersion situations. Ann. Inst. Statist. Math., 50(3), 567-585. https://doi.org/10.1023/A:1003585714207

Consul, P. C. (1989). Generalized Poisson distributions. Marcel Dekker, New York.

Daley, D. J., \& Maindonald, J. H. (1989). A unified view of models describing the avoidance of superparasitism. IMA Journal of Mathematics Applied in Biology and Medicine, 6, 161-78. https://doi.org/10.1093/imammb/6.3.161

Dimitrov, B., \& Kolev, N. (2000). Beta transformation. Beta type self-decomposability and related characterizations. Brazilian Journal of Probability and Statistics, 14, 123-140.

Geenwood, M., \& Yule, G. U. (1920). An inquiry into the nature of frequency distributions representative of multiple happenings with particular referee to the occurence of multiple attacks of disease or of repeated accidents. J. Roy. Statist. Soc., A 83, 255-279. https://doi.org/10.2307/2341080

Gupta, R. C., \& Ong, S. H. (2005). Analysis of long-tailed count data by Poisson mixtures. Communications in StatisticsTheory and Methods, 34, 557-573. https://doi.org/10.1081/STA-200052144

Haight, F. A. (1967). Handbook of the Poisson distribution. Wiley, New York.

Johnson, N. L., Kotz, S., \& Kemp, A. W. (2005). Univariate discrete distributions (Third Edition). John Wiley \& Sons, New York. https://doi.org/10.1002/0471715816

Jørgensen, B. (1997). The Theory of Dispersion Models. Chapman \& Hall, London.

Kendall, M. G., \& Stuart, A. (1979). The advanced theory of statistics. Macmillan, New York.

Kendall, M. G. (1961). Natural law in social sciences. Journal of the Royal Statistical Society Series A, 124, 1-19. https://doi.org/10.2307/2984139

Kokonendji, C. C., Mizère, D., \& Balakrishnan, N. (2008). Connections of the Poisson weight function to overdispersion and underdispersion. Journal of Statitical Planning and inference, 138, 1287-296. https://doi.org/10.1016/j.jspi.2007.05.028

Louzayadio, C. G. (2015). Decomposition of a weighted Poisson law into a convex combination of dual laws and Estimators comparison of the Poisson canonical parameter (Ph.D. thesis). Marien NGOUABI University, Congo.

Louzayadio, C. G., Mizère, D., \& Makany, R. A. (2013). Estimators comparison of the Poisson canonical parameter and mean dual distribution. Far East Journal of Theoretical Statistics, 44(2), 145-164.

Mizère, D. (2006). Contributions to the Modeling and Statistical Analysis of count Data (Ph.D. thesis). University of Pau, France.

Mizère, D., Louzayadio, C. G., Bidounga, R., \& Kissita, G. (2013). Decomposition of a weighted Poisson law into a 
convex combination of dual laws. Afrika Statistika, 8, 583-594.

Morgan, R. W. (1975). Some stochastic models to describe the fertilization of an egg. Applied Statistics, 24, 137-38. https://doi.org/10.2307/2346713

Morgan, B. J. T. (1982). Modelling polyspermy. Biometrics, 38, 885-98. https://doi.org/10.2307/2529869

Nelder, J. A., \& Mead, R. (1965). A simplex method for function minimization. The Computer Journal, 7(4), 308-313. https://doi.org/10.1093/comjn1/7.4.308

Patil, G. P. (2002). Weighted distributions. In Encyclopedia of Environmetrics(eds A.H. ElShaarawi and W. W. Piegorrsch). (Vol.4, pp. 2369-2377). Chichester: John Wiley \& Sons. https://doi.org/10.1002/9781118445112.stat07359

Patil, G. P., \& Rao, C. R. (1978). Weighted distributions and size-biased sampling with applications to widlife populations and human families. Biometrics, 34, 179-184. https://doi.org/10.2307/2530008

Patil, G. P., Rao, C. R., \& Ratnaparkhi, M. V. (1986). On discrete weighted distributions and their use in model choice for observed data. Communications in Statistics Theory and Methods, 15, 907-918. https://doi.org/10.1080/03610928608829159

Rao, C. R. (1965). On discrete distributions arising out of methods of ascertainment. Sankhyā Ser. A, 311-324.

Ridout, M. S. \& Besbeas, P. (2004). An empirical model for underdispersed count data. Statistical Modelling, 4, 77-89. https://doi.org/10.1191/1471082X04st064oa

Saporta, G. (2006). Probabilits, analyse des donnes et statistique. Editions Technip.

Shmueli, G., Minka, T. P., Kadane, J. B., Borles, S. \& Batwright, P. (2005). A useful distribution for fitting discrete data : revival of the Conway-Maywell Poisson distribution. Appl. Statist, 54(1), 127-142. https://doi.org /10.1111/j.14679876.2005.00474.x

\section{Copyrights}

Copyright for this article is retained by the author(s), with first publication rights granted to the journal.

This is an open-access article distributed under the terms and conditions of the Creative Commons Attribution license (http://creativecommons.org/licenses/by/4.0/). 